\title{
Ultrasonic Guided Waves in Piezoelectric Layered Composite with Different Interfacial Properties
}

\author{
Xiao Chen ${ }^{1,2}$ \\ ${ }^{1}$ Jiangsu Key Laboratory of Meteorological Observation and Information Processing, Nanjing University of Information Science and \\ Technology, Nanjing 210044, China \\ ${ }^{2}$ School of Electronic and Information Engineering, Nanjing University of Information Science and Technology, Nanjing 210044, China
}

Correspondence should be addressed to Xiao Chen, rainofsun@netease.com

Received 14 June 2011; Accepted 20 August 2011

Academic Editor: Wen-Hua Sun

Copyright (c) 2011 Xiao Chen. This is an open access article distributed under the Creative Commons Attribution License, which permits unrestricted use, distribution, and reproduction in any medium, provided the original work is properly cited.

\begin{abstract}
Combining the propagation model of guided waves in a multilayered piezoelectric composite with the interfacial model of rigid, slip, and weak interfaces, the generalized dispersion characteristic equations of guided waves propagating in a piezoelectric layered composite with different interfacial properties are derived. The effects of the slip, weak, and delamination interfaces in different depths on the dispersion properties of the lowest-order mode ultrasonic guided wave are analyzed. The theory would be used to characterize the interfacial properties of piezoelectric layered composite nondestructively.
\end{abstract}

\section{Introduction}

The acoustic wave propagation in piezoelectric materials has received considerable attention from engineering and scientific communities involved in nondestructive evaluation and transducer design. Numerous ultrasonic devices using piezoelectric materials are currently being developed for a variety of applications, such as SAW filters, ultrasonic transducers, and a variety of physical, chemical, and biological sensors $[1,2]$. In aerospace and other structural applications, piezoelectric layered smart or intelligent composites are being used [3]. Many of these applications involve layered structures made up of piezoelectric materials.

Layered composite materials are usually made of different materials. Very thin adhesive interfacial layer serves as the bonding between adjacent layers. Some imperfections, such as cracks and small voids, often emerge at the interface and contribute to the fatigue of the composite. The interfacial property between layers is one of the key factors that determine the structural stability of composites. It is, therefore, very important to characterize the adhesive layer quality, which has been a main topic in nondestructive testing and evaluation of composite materials [4-8].

Ultrasonic wave is a mechanical wave, which reflects the elastic properties of the medium. The interfacial mechanical property is closely related to the coupling strength between layers in the structure and the level of acoustic impedance match between the bonding layer and the piezoelectric material. Because of its deep penetration ability, ultrasonic technique is among the most promising nondestructive evaluation methods for the interfacial layer characterization. There are some papers considering the wave propagation in this structure with perfect interface, that is, the stress and displacement across the interface are continuous [9-11].

This paper is concerned with the guided waves propagating in piezoelectric layered composite with different interfacial properties. First, the transfer matrix of the piezoelectric layer is derived. Then, based on the model of piezoelectric layered composite, the general dispersion equations of the guided waves propagating in it with rigid, slip, and weak interfaces are presented by using the transfer matrix techniques. Numerical analysis has been performed on typical Y-cut, Z-propagating lithium niobate multi-layered composite with slip, weak, and delamination interface in different depth. It is shown that the interface property and the interfacial defect depth have strong effect on the guided wave phase velocity. The theoretical work in this paper is intended to provide fundamental principles in nondestructive testing and evaluation of the interface of piezoelectric layered composite. 


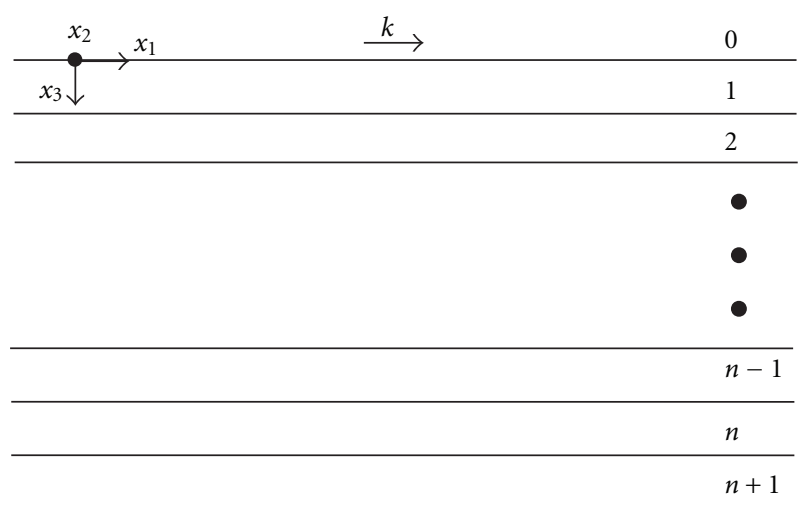

FIGURE 1: Labeling system and coordinate system of a piezoelectric multi-layered composite.

\section{Transfer Matrix of Piezoelectric Layer}

The transfer matrix method has long been used in modeling multi-layered structures [12]. The transfer matrix expresses the relationship of characterized parameters between the top and the bottom interface of a layer. The order of transfer matrix is determined by parameters of the layer material. To a piezoelectric layer, the transfer function consists of an $8 * 8$ matrix. Figure 1 shows the labeling system used in this paper. The waves propagate along the $x_{1}$ direction of a piezoelectric layer with its poling direction along $x_{3}$. We consider a two-dimensional problem assuming all field components are constant in the $x_{2}$ direction.

In the piezoelectric plate, the coupled wave equations using the quasistatic approximation are

$$
\begin{gathered}
\rho \frac{\partial^{2} u_{j}}{\partial t^{2}}-c_{i j k l} \frac{\partial^{2} u_{k}}{\partial x_{i} x_{l}}-e_{k i j} \frac{\partial^{2} \phi}{\partial x_{i} x_{k}}=0, \\
e_{i k l} \frac{\partial^{2} u_{k}}{\partial x_{i} x_{l}}-\varepsilon_{i k} \frac{\partial^{2} \phi}{\partial x_{i} x_{k}}=0,
\end{gathered}
$$

in which $\rho$ is the density of material, $u_{k}$ is the particle displacement vector, and $\phi$ is the electric potential. $c_{i j k l}$, $e_{i j k}$, and $\varepsilon_{i k}$ are, respectively, the tensor components of the elastic stiffness measured at constant electric field, the piezoelectric constant, and the dielectric permittivity measured at constant strain. The subscripts $i, j, k$, and $l$ range over the values 1,2 , and 3 , and the summation convention on repeated subscripts is employed. The solution of the form is assumed as

$$
\begin{aligned}
& u_{j}=\alpha_{j} \exp \left(i k b x_{3}\right) \exp \left[i k\left(x_{1}-v t\right)\right], \\
& \phi=\alpha_{4} \exp \left(i k b x_{3}\right) \exp \left[i k\left(x_{1}-v t\right)\right],
\end{aligned}
$$

where $b$ is the attenuation coefficient. Substituting (2) into (1), we obtain

$$
\left[\Gamma_{i j}-\delta_{i j}^{\prime} \rho v^{2}\right]\left[\alpha_{j}\right]=0
$$

where $v$ is the phase velocity of the wave. The symmetric matrix $\Gamma$ is given by

$$
\begin{gathered}
\Gamma_{i j}=c_{3 i 3 j} b^{2}+\left(c_{1 i 3 j}+c_{3 i 1 j}\right) b+c_{1 i 1 j}, \\
\Gamma_{i 4}=e_{3 i 3} b^{2}+\left(e_{1 i 3}+e_{3 i 1}\right) b+e_{1 i 1}, \\
\Gamma_{44}=-\left[\varepsilon_{33} b^{2}+\left(\varepsilon_{13}+\varepsilon_{31}\right) b+\varepsilon_{11}\right] .
\end{gathered}
$$

Equation (3) has a nontrivial solution only if

$$
\left|\Gamma_{i j}-\delta_{i j}^{\prime} \rho v^{2}\right|=0
$$

For every value of $v,(5)$ has eight eigenvalues of $b$. There are also eight four-component eigenvectors corresponding to the eight eigenvalues. Thus, the general solution can be written as

$$
\begin{aligned}
u_{j}= & \left\{\sum_{n} C_{n} \alpha_{j}^{(n)} \exp \left(i k b^{(n)} x_{3}\right)\right\} \\
& \times \exp \left[i k\left(x_{1}-v t\right)\right], \\
\phi= & \left\{\sum_{n} C_{n} \alpha_{4}^{(n)} \exp \left(i k b^{(n)} x_{3}\right)\right\} \\
& \times \exp \left[i k\left(x_{1}-v t\right)\right],
\end{aligned}
$$

where $C_{n}$ is the weighting coefficients.

The normal components of stress and electric displacement of piezoelectric materials are

$$
\begin{gathered}
\sigma_{3 j}=c_{3 j k l}\left(\frac{\partial u_{k}}{\partial x_{l}}\right)+e_{k 3 j}\left(\frac{\partial \phi}{\partial x_{k}}\right), \\
D_{3}=e_{3 k l}\left(\frac{\partial u_{k}}{\partial x_{l}}\right)-\varepsilon_{3 k}\left(\frac{\partial \phi}{\partial x_{k}}\right) .
\end{gathered}
$$

Assume that the displacement, stress, electric displacement, and potential are known at the top interface of the plate. According to (6) and (7), the weighting coefficients can be written as

$$
\left[\begin{array}{c}
C_{1} \\
C_{2} \\
C_{3} \\
C_{4} \\
C_{5} \\
C_{6} \\
C_{7} \\
C_{8}
\end{array}\right]=[M]_{\text {top }}^{-1}\left[\begin{array}{c}
u_{1} \\
u_{2} \\
u_{3} \\
\sigma_{31} \\
\sigma_{32} \\
\sigma_{33} \\
D_{3} \\
\phi
\end{array}\right]_{\text {top }}
$$


At the bottom interface, the displacement, stress, electric displacement, and potential are

$$
\left[\begin{array}{c}
u_{1} \\
u_{2} \\
u_{3} \\
\sigma_{31} \\
\sigma_{32} \\
\sigma_{33} \\
D_{3} \\
\phi
\end{array}\right]_{\text {bottom }}=[M]_{\text {bottom }} \cdot[M]_{\text {top }}^{-1} \cdot\left[\begin{array}{c}
u_{1} \\
u_{2} \\
u_{3} \\
\sigma_{31} \\
\sigma_{32} \\
\sigma_{33} \\
D_{3} \\
\phi
\end{array}\right]_{\text {top }}
$$

The matrix product in this equation now relates the characterized parameters between the top and the bottom interface of a piezoelectric layer and is defined as the transfer matrix $[P]$,

$$
[M]_{\text {bottom }}[M]_{\text {top }}^{-1}=[P] .
$$

\section{Wave Equations in Piezoelectric Layered Composite}

The model and the coordinate system of the piezoelectric layered composite are shown in Figure 1. Waves propagate along the $x_{1}$ direction. In the transfer matrix method, the intermediate interfaces are eliminated so that the fields in all layers are described solely in terms of external boundary conditions. The displacement, stress, electric displace, and potential must be continuous across a rigid interface between two layers;

$$
\left[\begin{array}{c}
u_{1} \\
u_{2} \\
u_{3} \\
\sigma_{31} \\
\sigma_{32} \\
\sigma_{33} \\
D_{3} \\
\phi
\end{array}\right]_{j+1, \text { top }}=[P]_{j}\left[\begin{array}{c}
u_{1} \\
u_{2} \\
u_{3} \\
\sigma_{31} \\
\sigma_{32} \\
\sigma_{33} \\
D_{3} \\
\phi
\end{array}\right]_{j, \text { top }}
$$

This process can be continued layer by layer, resulting in the follwing equation;

$$
\left[\begin{array}{c}
u_{1} \\
u_{2} \\
u_{3} \\
\sigma_{31} \\
\sigma_{32} \\
\sigma_{33} \\
D_{3} \\
\phi
\end{array}\right]_{n, \text { bottom }}=[Q]\left[\begin{array}{c}
u_{1} \\
u_{2} \\
u_{3} \\
\sigma_{31} \\
\sigma_{32} \\
\sigma_{33} \\
D_{3} \\
\phi
\end{array}\right]_{1, \text { top }}
$$

where $n$ is the last layer and $[Q]$ is the system matrix consisting of the matrix product of layer matrices;

$$
[Q]=[P]_{1} \cdot[P]_{2} \cdots[P]_{n-1} \cdot[P]_{n} .
$$

The wave must satisfy the appropriate electrical and mechanical boundary conditions on both surfaces of the composite. The top and bottom of composite are free. So the normal stresses at the extreme interfaces (top and bottom) must be zero. Then the mechanical boundary conditions are

$$
\begin{aligned}
& {\left[\sigma_{31}\right]_{n, \text { bottom }}=\left[\sigma_{31}\right]_{1, \text { top }}=0,} \\
& {\left[\sigma_{32}\right]_{n, \text { bottom }}=\left[\sigma_{32}\right]_{1, \text { top }}=0,} \\
& {\left[\sigma_{33}\right]_{n, \text { bottom }}=\left[\sigma_{33}\right]_{1, \text { top }}=0 .}
\end{aligned}
$$

The metallization on plate surfaces is assumed to consist of a perfectly conducting film of negligible thickness. Thus, the presence of the metal film does not alter the mechanical boundary conditions. However, the electrical boundary conditions vary according to whether it is open (unmetallized) or shorted (metallized). We consider the case where both surfaces are open circuit condition. The electrical boundary conditions are

$$
\begin{gathered}
{\left[D_{3}\right]_{n, \text { bottom }}=k \varepsilon_{0}[\phi]_{n, \text { bottom }},} \\
{\left[D_{3}\right]_{1, \text { top }}=-k \varepsilon_{0}[\phi]_{1, \text { top }}}
\end{gathered}
$$

where $\varepsilon_{0}$ is the dielectric constant of the vacuum. According to (14)-(17), (12) can be written as

$$
\begin{aligned}
& {[A]\left[\begin{array}{c}
u_{1} \\
u_{2} \\
u_{3} \\
\phi
\end{array}\right]_{1, \text { top }}=0} \\
& {[A]=\left[\begin{array}{cccc}
Q_{41} & Q_{42} & Q_{43} & Q_{48}\left(1+k \varepsilon_{0}\right) \\
Q_{51} & Q_{52} & Q_{53} & Q_{58}\left(1+k \varepsilon_{0}\right) \\
Q_{61} & Q_{62} & Q_{63} & Q_{68}\left(1+k \varepsilon_{0}\right) \\
Q_{71}-k \varepsilon_{0} Q_{81} & Q_{72}-k \varepsilon_{0} Q_{82} & Q_{73}-k \varepsilon_{0} Q_{83} & \left(Q_{78}-k \varepsilon_{0} Q_{88}\right)\left(1+k \varepsilon_{0}\right)
\end{array}\right] .}
\end{aligned}
$$


For this equation to be satisfied, the submatrix must be singular,

$$
|A|=0 .
$$

Equation (20) is the dispersion equation of the piezoelectric layered composite with rigid interfacial condition.

If there is a slip interface between the $j$ th and the $(j+1)$ th layers, the interface can only transfer normal displacement and stress. The normal displacement and stress of two layers are continuous, and the shear stresses are zero. So the interfacial boundary conditions are

$$
\begin{gathered}
{\left[u_{3}\right]_{j, \text { bottom }}=\left[u_{3}\right]_{j+1, \text { top }},} \\
{\left[\sigma_{31}\right]_{, j \text {,bottom }}=0,} \\
{\left[\sigma_{31}\right]_{j+1, \text { top }}=0,} \\
{\left[\sigma_{32}\right]_{j, \text { bottom }}=0,} \\
{\left[\sigma_{32}\right]_{j+1, \text { top }}=0,} \\
{\left[\sigma_{33}\right]_{j, \text { bottom }}=\left[\sigma_{33}\right]_{j+1, \text { top }},} \\
{\left[D_{3}\right]_{j, \text { bottom }}=\left[D_{3}\right]_{j+1, \text { top }},} \\
{[\phi]_{j, \text { bottom }}=[\phi]_{j+1, \text { top }}}
\end{gathered}
$$

The other interfaces of the composite are all rigid, therefore

$$
\left[\begin{array}{c}
u_{1} \\
u_{2} \\
u_{3} \\
\sigma_{31} \\
\sigma_{32} \\
\sigma_{33} \\
D_{3} \\
\phi
\end{array}\right]_{n, \text { bottom }}=[D]\left[\begin{array}{c}
u_{1} \\
u_{2} \\
u_{3} \\
\sigma_{31} \\
\sigma_{32} \\
\sigma_{33} \\
D_{3} \\
\phi
\end{array}\right]_{j+1, \text { top }},
$$

where

$$
\begin{gathered}
{[D]=[P]_{j+1} \cdot[P]_{j+2} \ldots[P]_{N-1} \cdot[P]_{n},} \\
{\left[\begin{array}{c}
u_{1} \\
u_{2} \\
u_{3} \\
\sigma_{31} \\
\sigma_{32} \\
\sigma_{33} \\
D_{3} \\
\phi
\end{array}\right]_{j, \text { bottom }}=[E]\left[\begin{array}{c}
u_{1} \\
u_{2} \\
u_{3} \\
\sigma_{31} \\
\sigma_{32} \\
\sigma_{33} \\
D_{3} \\
\phi
\end{array}\right]_{1, \text { top }},}
\end{gathered}
$$

where

$$
[E]=[P]_{1} \cdot[P]_{2} \ldots[P]_{j-1} \cdot[P]_{j} .
$$

By combining (22)-(24) with (21), the dispersion equation of the piezoelectric layered composite with a slip interfacial condition becomes

$$
\left|\begin{array}{llll}
D_{41} & D_{42} & F_{11} & F_{12} \\
D_{51} & D_{52} & F_{21} & F_{22} \\
D_{61} & D_{62} & F_{31} & F_{32} \\
D_{71}^{*} & D_{72}^{*} & F_{41} & F_{42}
\end{array}\right|=0
$$

where

$$
\begin{aligned}
{\left[\begin{array}{ll}
F_{11} & F_{12} \\
F_{21} & F_{22} \\
F_{31} & F_{32} \\
F_{41} & F_{42}
\end{array}\right]=} & {\left[\begin{array}{llll}
D_{43} & D_{46} & D_{47} & D_{48} \\
D_{53} & D_{56} & D_{57} & D_{58} \\
D_{63} & D_{66} & D_{67} & D_{68} \\
D_{73}^{*} & D_{76}^{*} & D_{77}^{*} & D_{78}^{*}
\end{array}\right] } \\
& \cdot\left[\begin{array}{llll}
E_{31} & E_{32} & E_{33} & E_{38}^{*} \\
E_{61} & E_{62} & E_{63} & E_{68}^{*} \\
E_{71} & E_{72} & E_{73} & E_{78}^{*} \\
E_{81} & E_{82} & E_{83} & E_{88}^{*}
\end{array}\right] \cdot\left[\begin{array}{cc}
1 & 0 \\
0 & 1 \\
G_{11} & G_{12} \\
G_{21} & G_{22}
\end{array}\right], \\
{\left[\begin{array}{ll}
G_{11} & G_{12} \\
G_{21} & G_{22}
\end{array}\right]=} & {\left[\begin{array}{lll}
-E_{43} & -E_{48}^{*} \\
-E_{53} & -E_{58}^{*}
\end{array}\right]^{-1} \cdot\left[\begin{array}{ll}
E_{41} & E_{42} \\
E_{51} & E_{52}
\end{array}\right] . }
\end{aligned}
$$

The rows 1 to 6 and 8 of the matrix $[D]^{*}$ are the same as those of matrix $[D]$. The row 7 of matrix $[D]^{*}$ satisfies

$$
D_{7 j}^{*}=D_{7 j}-k \varepsilon_{0} D_{8 j} .
$$

The columns 1 to 6 and 8 of matrix $[E]$ are the same as those of matrix $[E]^{*}$. The column 7 of the matrix $[E]$ satisfies

$$
E_{j 7}^{*}=E_{j 7}+k \varepsilon_{0} E_{j 8} .
$$

If there is a weak interface between the $j$ th and the $(j+$ 1)th layers, the "spring" model can be used to characterize the interface property $[13,14]$. So the interfacial boundary conditions are

$$
\begin{aligned}
& {\left[\sigma_{33}\right]_{j, \text { bottom }}=\left[\sigma_{33}\right]_{j+1, \text { top }}=K_{n}\left(\left[u_{3}\right]_{j, \text { bottom }}-\left[u_{3}\right]_{j+1, \text { top }}\right),} \\
& {\left[\sigma_{31}\right]_{j, \text { bottom }}=\left[\sigma_{31}\right]_{j+1, \text { top }}=K_{t 1}\left(\left[u_{1}\right]_{j, \text { bottom }}-\left[u_{1}\right]_{j+1, \text { top }}\right),} \\
& {\left[\sigma_{32}\right]_{j, \text { bottom }}=\left[\sigma_{32}\right]_{j+1, \text { top }}=K_{t 2}\left(\left[u_{2}\right]_{j, \text { bottom }}-\left[u_{2}\right]_{j+1, \text { top }}\right),}
\end{aligned}
$$

where $K_{n}, K_{t 1}$, and $K_{t 2}$ are the normal stiffness coefficient, the shear stiffness coefficient of the $x_{1}$-dimension and $x_{2}$ dimension, respectively.

The dispersion equation of the piezoelectric layered composite with a weak interfacial condition is given by

$$
\left|\begin{array}{cccc}
Z_{41} & Z_{42} & Z_{43} & Z_{48} \\
Z_{51} & Z_{52} & Z_{53} & Z_{58} \\
Z_{61} & Z_{62} & Z_{63} & Z_{68} \\
Z_{71} & Z_{72} & Z_{73} & Z_{78}
\end{array}\right|=0
$$




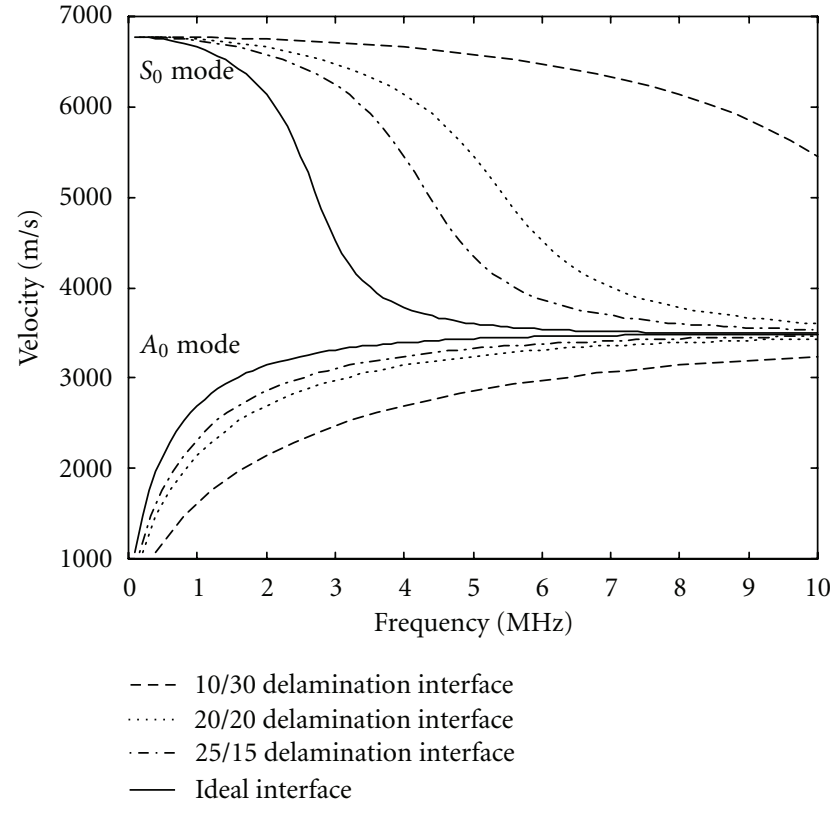

Figure 2: The $A_{0}$ mode dispersion curve of $\mathrm{YZ} \mathrm{LiNiO}_{3}$ multilayered plate with a delamination interface in different depth.

where

$$
[H]=\left[\begin{array}{cccccccc}
1 & 0 & 0 & -\frac{1}{K_{t 1}} & 0 & 0 & 0 & 0 \\
0 & 1 & 0 & 0 & -\frac{1}{K_{t 2}} & 0 & 0 & 0 \\
0 & 0 & 1 & 0 & 0 & -\frac{1}{K_{n}} & 0 & 0 \\
0 & 0 & 0 & 1 & 0 & 0 & 0 & 0 \\
0 & 0 & 0 & 0 & 1 & 0 & 0 & 0 \\
0 & 0 & 0 & 0 & 0 & 1 & 0 & 0 \\
0 & 0 & 0 & 0 & 0 & 0 & 1 & 0 \\
0 & 0 & 0 & 0 & 0 & 0 & 0 & 1
\end{array}\right],
$$

\section{Numerical Results and Discussion}

The analysis presented in the previous section has been applied to calculate the characteristics of the $A_{0}$ and $S_{0}$ modes propagating in a $\mathrm{Y}$-cut, $\mathrm{Z}$-propagating lithium niobate layered composite. The material constants of the $\mathrm{LiNiO}_{3}$ come from [15]. Since this paper deals with the effect of the interfacial properties of composite on the dispersion characteristics of the guided waves propagating, it is assumed that each layer has the same material, crystal type, cut direction, and thickness. The piezoelectric plate has 40 layers, and the thickness of each layer is $25 \mu \mathrm{m}$. In this paper, the structure with a defective interface is represented as $\mathrm{m} / \mathrm{n}$. The left and right number of the symbol "/" represent the number of layers on and under the defective interface.

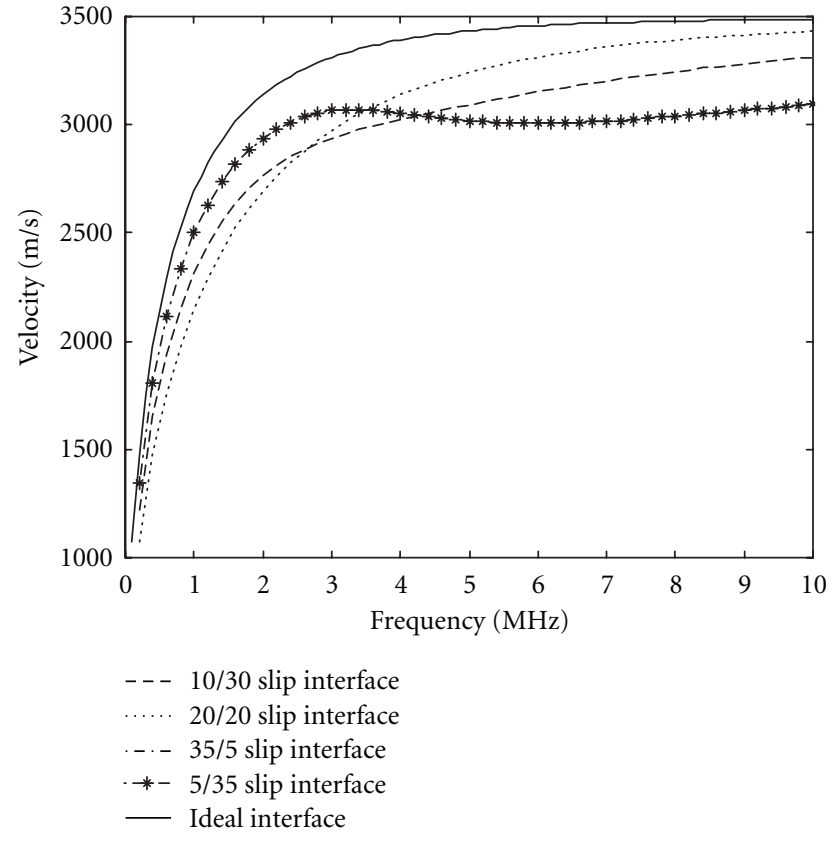

Figure 3: The $A_{0}$ mode dispersion curve of $\mathrm{YZ} \mathrm{LiNiO}_{3}$ multilayered plate with a slip interface in different depth.

Figure 2 shows the Lamb wave phase velocities of the $A_{0}$ and $S_{0}$ modes as a function of frequency with a delamination interface in different depth. The dispersion characteristic of $A_{0}$ and $S_{0}$ modes in an $\mathrm{YZ} \mathrm{LiNiO}_{3}$ plate looks very similar to that of the lowest order Lamb waves propagating in a plate of isotropic plate [16]. However, It should be noted that $A_{0}$ and $S_{0}$ modes in piezoelectric composite have all three components of particle displacement, that is, the waves are more general Lamb waves. As the frequency increases, the $A_{0}$ and $S_{0}$ mode velocities tend asymptotically to the SAW velocity. Further, it can be shown that the phase velocity of the $A_{0}$ mode increases when the depth of the delamination interface increases while the phase velocity of the $S_{0}$ mode decreases when the depth of the delamination interface increases. Figure 3 shows the Lamb wave phase velocity of the $A_{0}$ mode as a function of frequency with a slip interface in different depth. Except for the 20/20 structure, the phase velocity does not tend asymptotically to a constant velocity when the frequency increases. If the frequency less than 2.5 MHz is considered, the phase velocity of the Lamb wave decreases with the depth when it is less than one-half of the plate thickness, but increases with the depth when it is more than one-half of the plate thickness.

When the interface is weak, the stiffness $K_{n}$ and $K_{t i}$ are finite values. Figure 4 shows the Lamb wave phase velocity of the $A_{0}$ mode as a function of frequency with a weak interface in different depth. It is assumed that $K_{n}$ and $K_{t 2}$ are infinite, and that $K_{t 1}$ is $5 \times 10^{15} \mathrm{~N} / \mathrm{m}^{3}$. Like the condition of the slip interface, the phase velocity does not show saturation when the frequency increases, except for the 20/20 structure. From Figures 3 and 4, one can find that the dispersion characteristic with a weak interface is between that of a slip interface in 


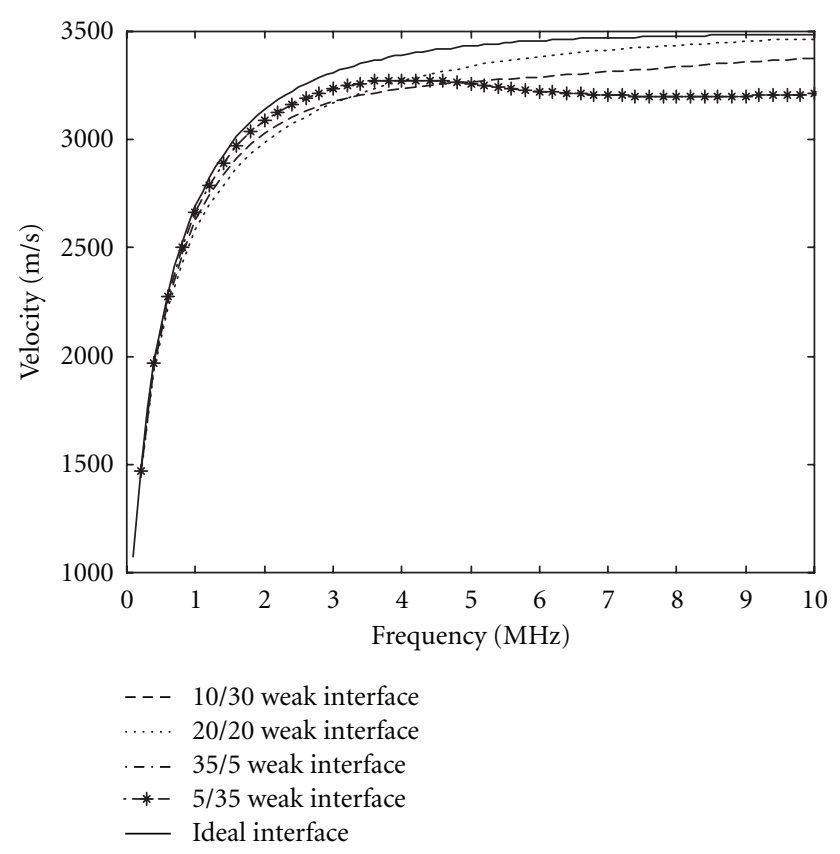

Figure 4: The $A_{0}$ mode dispersion curve of $\mathrm{YZ} \mathrm{LiNiO}_{3}$ multilayered plate with a weak interface in different depth.

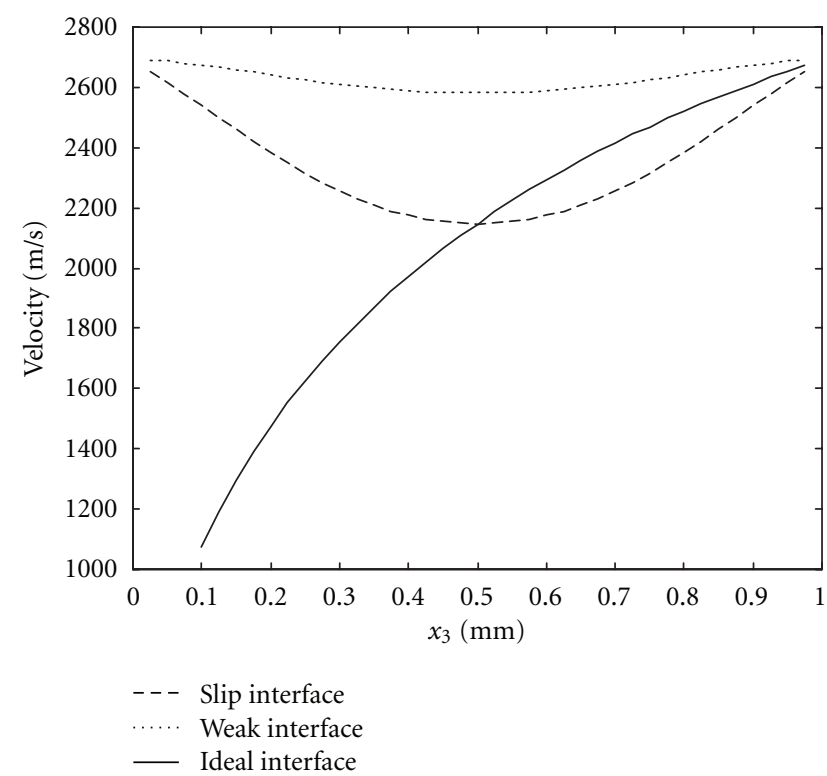

FIGURE 5: The phase velocity of $A_{0}$ mode versus the default depth with the $1 \mathrm{MHz}$ frequency.

the same depth and that of the ideal rigid interface. Furthermore, the dispersion characteristic with a weak interface in the central plate is the same as that of a slip interface.

Figure 5 shows the $A_{0}$ mode phase velocity versus the defect depth at $1 \mathrm{MHz}$. When the central interface of the composite has a delamination interface, the phase velocity is the same as that when it has a slip interface. When the depth of the delamination interface is less than half of the thickness of the composite, the phase velocity is less than that

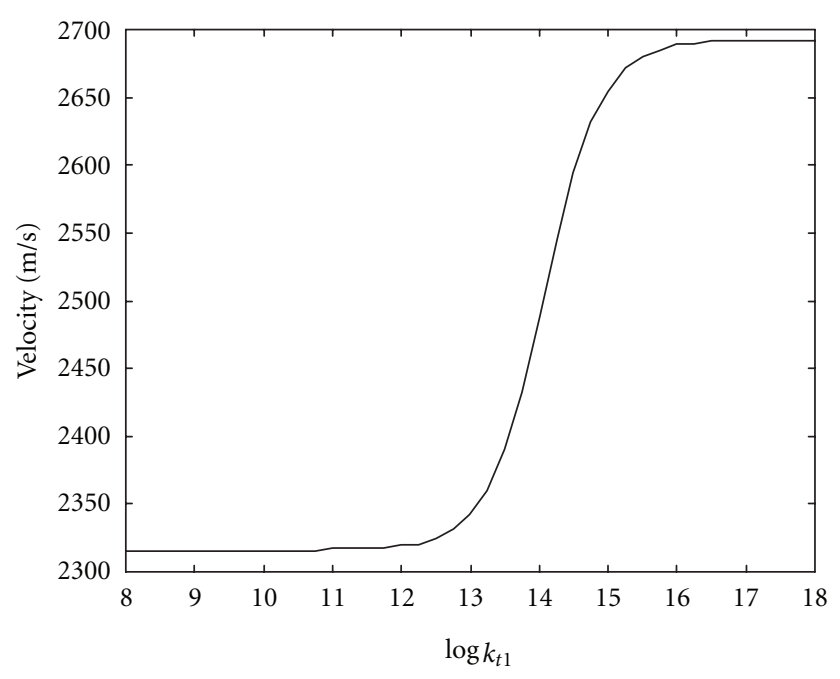

Figure 6: The effect of the interface stiffness constants $K_{t 1}\left(\mathrm{~N} / \mathrm{m}^{3}\right)$ on phase velocity of $A_{0}$ mode.

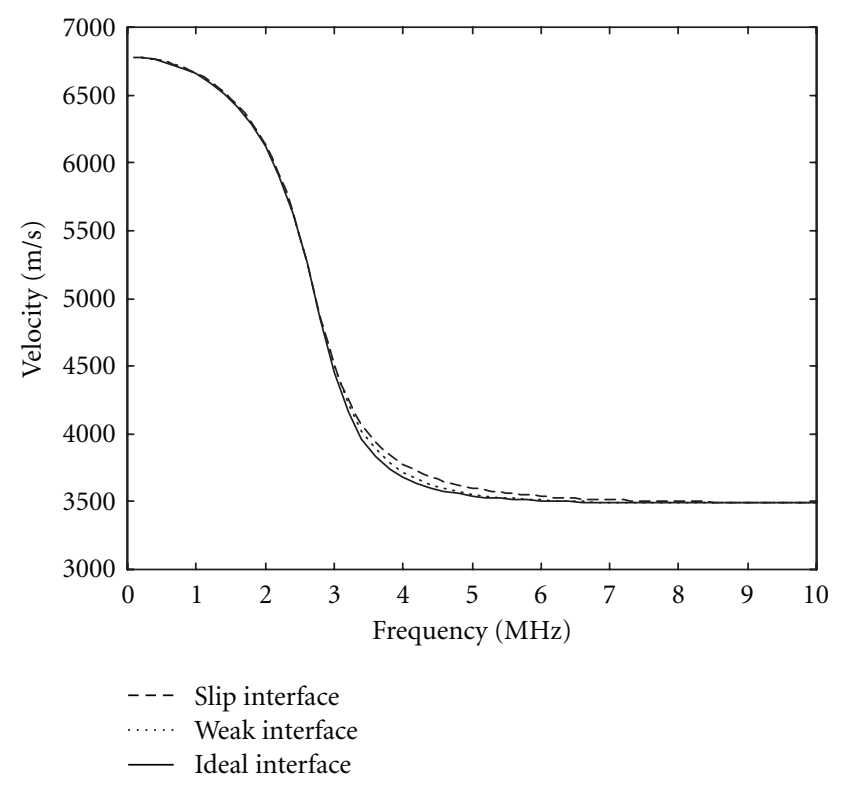

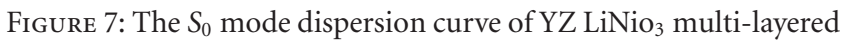
plate with a default interface in 10/30 structure.

of a slip interface in the same depth, and vice versa. The phase velocity with a delamination and slip interface is less than that with a weak interface in the same depth.

Figure 6 presents the effect of the interface stiffness constants $K_{t 1}$ on the phase velocity of the $A_{0}$ mode in the $10 / 30$ composite structure. In the calculation $f$ is equal to $1 \mathrm{MHz}$, and $K_{n}$ and $K_{t 2}$ are infinite. It reveals that the phase velocity of the $A_{0}$ mode increases with the increasing $K_{t 1}$. When $K_{t 1}$ approaches zero, the phase velocity approaches that of a composite with a slip interface. When $K_{t 1}$ approaches infinity, the phase velocity approaches to that of a composite with a rigid interface. Very large variation of 
the phase velocity of the $A_{0}$ mode can be seen when $K_{t 1}$ varies in the range of $10^{12}-10^{15} \mathrm{~N} / \mathrm{m}^{3}$.

Figure 7 shows the Lamb wave phase velocity of the $S_{0}$ mode as a function of frequency with a rigid, slip, and weak interface in the $10 / 30$ composite structure. Unlike $A_{0}$ mode, the interface properties have very little effect on the phase velocity of $S_{0}$ mode.

\section{Conclusions}

We have conducted a theoretical investigation of the velocity variation of guided waves propagating in piezoelectric multi-layered composite with different interfacial properties. General dispersion equations for a composite that contains rigid, slip, and weak interfaces are derived by using the transfer matrix technique. The phase velocities of the lowest antisymmetry and symmetry modes have been numerically computed on a YZ lithium niobate layered composite with slip, weak, and delamination interface in different depth. As the depth of the defective interface changes, the velocity of the lowest anti-symmetry mode changes drastically. But the velocity of the lowest symmetry mode is almost not effected by the interface characteristics. When the interface stiffness coefficient varies in the range of $10^{12}-10^{15} \mathrm{~N} / \mathrm{m}^{3}$, the velocity of the lowest anti-symmetry mode has the largest change.

\section{Acknowledgments}

This paper is supported by the National Natural Science Foundation of China (10904073), the Natural Science Research Project of Jiangsu Higher Education Institutions (10 KJB510011), and the Priority Academic Program Development of Jiangsu Higher Education Institutions.

\section{References}

[1] S. W. Wenzel and R. M. White, "A multisensor employing an ultrasonic Lamb-wave oscillator," IEEE Transactions on Electron Devices, vol. 35, no. 6, pp. 735-743, 1988.

[2] L.-F. Ge and J.-P. Shao, "Capacitive lamb wave transducers with multiple even-modes for biochemical detections," in 6th International Symposium on Precision Engineering Measurements and Instrumentation, vol. 7544 of Proceedings of SPIE, December 2010.

[3] H. Uyanik and Z. Mecitoğlu, "Active vibration control of a laminated composite plate subjected to blast load," Aircraft Engineering and Aerospace Technology, vol. 81, no. 4, pp. 308315, 2009.

[4] A. Velichko and P. D. Wilcox, "Modeling the excitation of guided waves in generally anisotropic multilayered media," Journal of the Acoustical Society of America, vol. 121, no. 1, pp. 60-69, 2007.

[5] Y. H. Kim, D. H. Kim, J. H. Han, and C. G. Kim, "Damage assessment in layered composites using spectral analysis and Lamb wave," Composites Part B: Engineering, vol. 38, no. 7-8, pp. 800-809, 2007.

[6] Zh. Su, L. Ye, and Y. Lu, "Guided Lamb waves for identification of damage in composite structures: a review," Journal of Sound and Vibration, vol. 295, no. 3-5, pp. 753-780, 2006.
[7] T. Kundu, A. Maji, T. Ghosh, and K. Maslov, "Detection of kissing bonds by Lamb waves," Ultrasonics, vol. 35, no. 8, pp. 573-580, 1998.

[8] W. Yang and T. Kundu, "Guided waves in multilayered plates for internal defect detection," Journal of Engineering Mechanics, vol. 124, no. 3, pp. 311-327, 1998.

[9] D. H. Cortes, S. K. Datta, and O. M. Mukdadi, "Elastic guided wave propagation in a periodic array of multi-layered piezoelectric plates with finite cross-sections," Ultrasonics, vol. 50, no. 3, pp. 347-356, 2010.

[10] M. Lam, E. Le Clézio, H. Amorín et al., "Acoustic wave transmission through piezoelectric structured materials," Ultrasonics, vol. 49, no. 4-5, pp. 424-431, 2009.

[11] B. Collet, "Recursive surface impedance matrix methods for ultrasonic wave propagation in piezoelectric multilayers," Ultrasonics, vol. 42, no. 1-9, pp. 189-197, 2004.

[12] M. J. S. Lowe, "Matrix techniques for modeling ultrasonic waves in multilayered media," IEEE Transactions on Ultrasonics, Ferroelectrics, and Frequency Control, vol. 42, no. 4, pp. 525-542, 1995.

[13] S. I. Rokhlin and Y. J. Wang, "Equivalent boundary conditions for thin orthotropic layer between two solids: reflection, refraction, and interface waves," Journal of the Acoustical Society of America, vol. 91, no. 4, pp. 1875-1887, 1992.

[14] X. Chen and M. Wan, "Guided waves in piezoelectric coating composite structure with weak interface," Chinese Journal of Acoustics, vol. 28, no. 4, pp. 363-367, 2003.

[15] B. Auld, Acoustic Field and Waves in Solids, part 2, John Wiley \& Sons, New York, NY, USA, 1973.

[16] I. V. Viktorov, Rayleigh and Lamb Waves, Plenum Press, New York, NY, USA, 1967. 

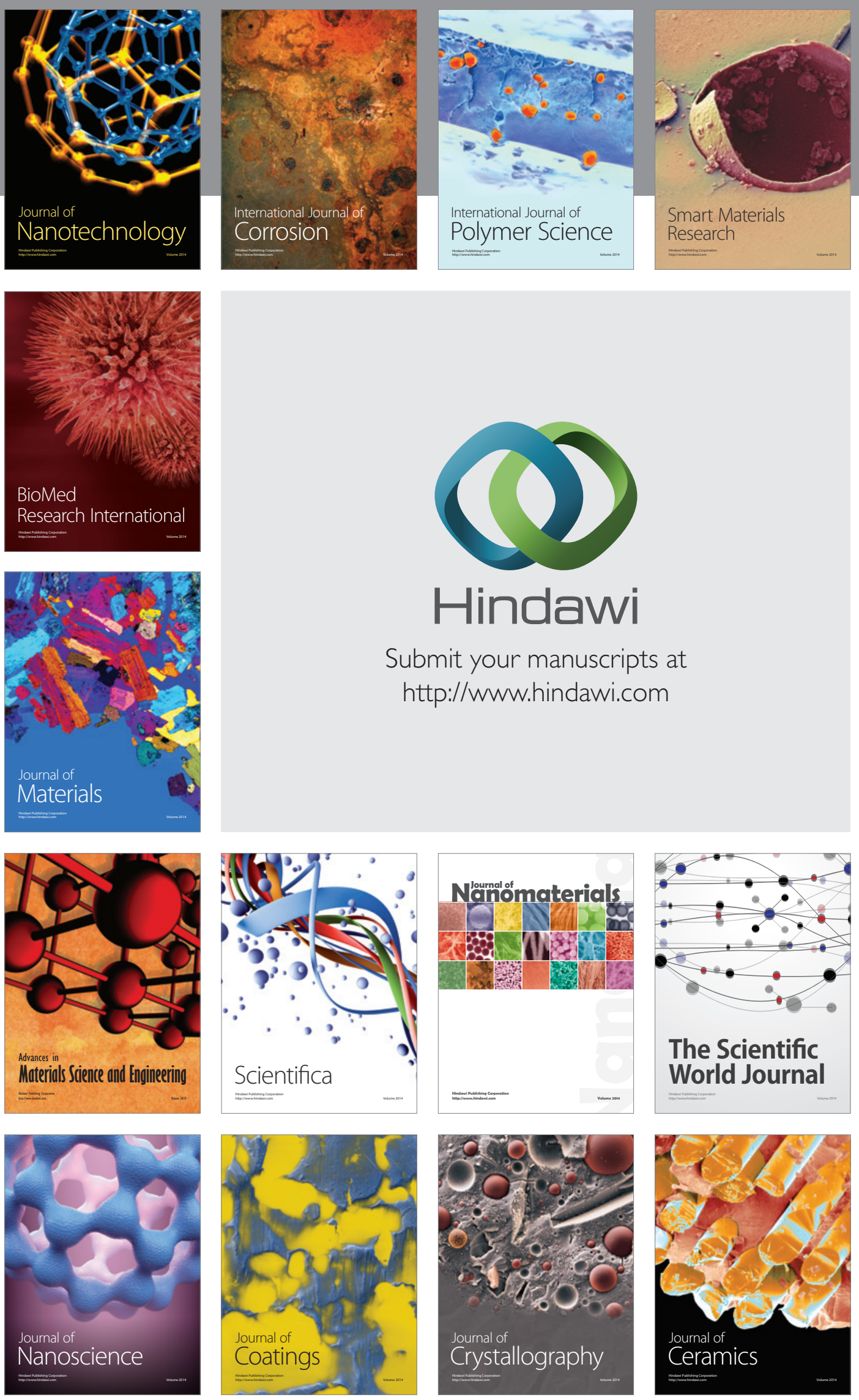

The Scientific World Journal

Submit your manuscripts at

http://www.hindawi.com

\section{World Journal}

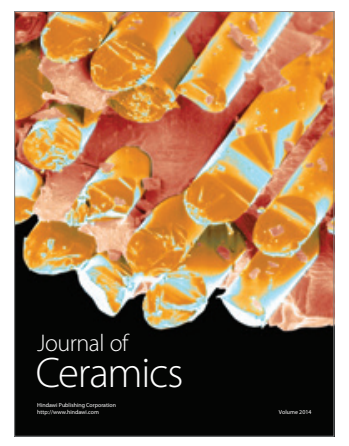

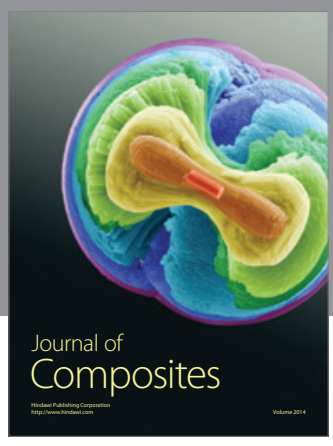
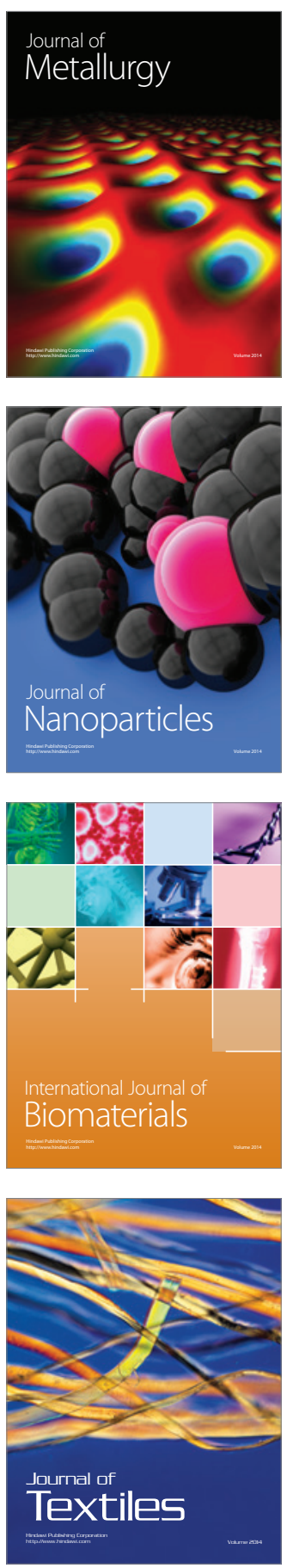